\title{
Adubos e coberturas orgânicas, fontes de nutrientes de liberação lenta na produção de acelga de colheitas múltiplas
}

\author{
Fertilizers and organic covers, slow release nutrient sources in the production of multiple harvest \\ Swiss chard
}

\section{Hipolito Murga-Orrillo1,2*, Jhon Maber Irigoín-Aguilar ${ }^{3}$, Sharmely Hilares-Vargas ${ }^{1}$, Ricardo Manuel Bardales-Lozano ${ }^{4}$ e Francisco De Almeida Lobo ${ }^{1}$}

\author{
${ }^{1}$ Universidade Federal de Mato Grosso, Cuiabá, MT, Brasil. *Autor para correspondência: leohmurga@gmail.com. \\ 2Universidad Nacional Autónoma de Chota, Chota, Perú. \\ 3Universidad Nacional de Cajamarca, Cajamarca, Perú. \\ ${ }^{4}$ Instituto de Investigaciones de la Amazonia Peruana, Pucallpa, Perú
}

Submissão: 05/01/2019 / Aceite: 05/04/2019

\begin{abstract}
RESUMO
Objetivou-se avaliar o desempenho de coberturas e adubos orgânicos na estabilidade produtiva da acelga. O delineamento experimental foi de blocos casualizados, em arranjo fatorial e em parcelas subdivididas; usando húmus de minhoca (A1), guano de ilha (A2), e húmus de esterco de porquinho-daíndia (A3) como adubos, serragem de pinheiro-de-folhas-pêndulas (C1), casca de arroz (C2), e palha de aveia (C3) como coberturas, para três colheitas sucessivas. Avaliou-se, porcentagem de emergência (E), comprimento de folhas (CF), massa fresca de folhas (MFF) e massa seca de folhas (MSF). Nos resultados, as coberturas que propiciaram os maiores valores foram a C2 e C3 com 43,1 e 42,7 cm no CF, 209,9 e 215,5 g no MFF, respectivamente; nos adubos, o A3 proporcionou os maiores valores com 92,0\% na E, 44,2 cm no CF, 231,6 g no MFF, e $154 \mathrm{~g}$ no MSF. As coberturas de C2 e C3 e, o adubo de A3 condicionaram os melhores desempenhos no CF e MFF em relação aos outros tratamentos; os rendimentos mantiveram-se na $1^{\underline{a}}$ e $2^{a}$ colheita baixando significativamente na $3^{a}$ colheita; o húmus de esterco de porquinho-da-índia apresenta caraterísticas nutricionais destacáveis para seu uso na horticultura.
\end{abstract}

PALAVRAS-CHAVE: húmus, guano de ilha, esterco de porquinho-da-índia.

\begin{abstract}
The objective of this study was to evaluate the performance of organic coverage and fertilizers on the production stability of Swiss chard. The experimental design was randomized blocks, in factorial arrangement and subdivided plots; using earthworm humus (A1), island guano (A2), and guinea pig manure (A3) as fertilizers, pine-leaf-sawdust (C1), rice husk (C2), and oat straw (C3) as covering, for three successive harvests. Percentage of emergence (E), length leaf (CF), fresh mass (MFF) weight, and dry mass (MSF) weight were evaluated. In the results, the coverages that provided the highest values were C2 and C3 with 43.1 and $42.7 \mathrm{~cm}$ in the CF, 209.9 and $215.5 \mathrm{~g}$ in the MFF, respectively; in fertilizers, A3 provided the highest values with $92.0 \%$ in $\mathrm{E}, 44.2 \mathrm{~cm}$ in CF, $231.6 \mathrm{~g}$ in MFF, and $154 \mathrm{~g}$ in MSF. The C2 and C3 coverages and the A3 fertilizer conditioned the best performances in the CF and MFF in relation to the other treatments; the yields remained in the 1st and 2nd harvest, dropping significantly in the 3rd harvest; humus from guinea pig manure presented detachable nutritional characteristics for use in horticulture.
\end{abstract}

KEYWORDS: humus, island guano, guinea pig manure.

A acelga (Beta vulgaris L. var. cicla) é uma hortaliça de folha de múltiplas colheitas. As folhas são fonte de proteínas, vitaminas, carotenoides e minerais, com baixo conteúdo de calorias (DZIDA \& PITURA 2008, KOŁOTA et al. 2010). Esta cultura propaga-se por semente, de forma direta ou indireta. Entre os 70 a 90 dias despois da semeadura (DDS), as folhas mostram-se verde-escuras e suculentas, e os pecíolos brancos, indicando o momento do início de colheita destas folhas, sendo que daí por diante realizam-se colheitas sucessivas por mais de dois meses, mas os rendimentos diminuem progressivamente neste 
período.

É escassa a informação de respostas produtivas da acelga a coberturas e adubação orgânica. As coberturas orgânicas usam-se como estratégia para disponibilizar nutrientes de maneira lenta (MURGAORRILLO et al. 2016a), do mesmo modo, se em paralelo usa-se adubos orgânicos, as variações dos rendimentos entre colheitas poderiam ser mínimas nesta cultura.

$\mathrm{Na}$ agricultura andina peruana, os adubos orgânicos tradicionais são o guano de ilha (esterco e restos descompostos de aves marinhas) e o húmus de esterco de porquinho-da-índia (esterco descomposto de Cavia porcellus), com bons resultados. A partir do século XX, inserta-se a vermicultura e também o húmus de minhoca. Agora, quando se trata de restos de colheita, geralmente estes são queimados ao implantar a seguinte safra; podendo-se usar como coberturas e aproveitar assim seus benefícios para o solo e a cultura.

Os adubos orgânicos melhoram a fertilidade física, química e biológica do solo (RODRIGUES \& CASALI 1998, COMESE et al. 2009, FERREIRA et al. 2013, MUÑOZ et al. 2015, XIUKANG et al. 2015, HERNÁNDEZ et al. 2016), beneficiando o crescimento das culturas, ao facilitar a disponibilidade de nutrientes e água (GONÇALVES et al. 2005), melhorando a produtividade com rendimentos equivalentes ou superiores aos obtidos convencionalmente (XIUKANG et al. 2015, MUÑOZ et al. 2015). Similares benefícios proporcionam as coberturas ao solo, dotando de nutrientes ao mineralizar-se a mediano prazo; mas, inicialmente protegem de infestações de plantas daninhas, da incidência da luz e amplitude térmica, melhorando as condições hídricas, químicas, físicas e biológicas do solo (BORKERT et al. 2003, RESENDE et al. 2005, COSTA et al. 2007), visado a um melhor desenvolvimento fisiológico das culturas.

Com o exposto, surgem expectativas para usar coberturas e adubos orgânicos como fontes de nutrientes de liberação lenta, para culturas hortícolas de folhas de múltiplas colheitas. Neste trabalho objetivou-se avaliar o desempenho de coberturas e adubos orgânicos na estabilidade produtiva da acelga, para condições de Cajamarca, Perú.

O experimento foi conduzido no período de 15/10/2015 ao 25/03/2016 no Silvo Agropecuario-Facultad de Ciencias Agrarias (SA-FCA), campus da Universidad Nacional de Cajamarca (UNC), a $07^{\circ} 29^{\prime} 45^{\prime \prime} \mathrm{S}$ e $78^{\circ} 10^{\prime} 12^{\prime \prime} \mathrm{O}$, a $2670 \mathrm{~m}$ de altitude. O sítio tem um clima $\mathrm{Cw}$ - chuvoso temperado com inverno seco (KÖPPEN 1931), classificado segundo os dados climáticos da estação meteorológica Augusto Weberbauer, e os dados do clima para o período de condução do experimento se mostra na Tabela 1.

Tabela 1. Dados meteorológicos dos meses da condução do experimento. SA-FCA/UNC. Cajamarca, 2015/2016.

Table 1. Meteorological data from the months of the experiment. SA-FCA / UNC. Cajamarca, 2015/16.

\begin{tabular}{|c|c|c|c|c|c|}
\hline \multirow[t]{2}{*}{ Meses } & $\mathrm{T}$ & Ev & $\mathrm{Pp}$ & ETo & $\mathrm{HR}$ \\
\hline & $\ldots{ }^{\circ} \mathrm{C} \ldots$ & \multicolumn{3}{|c|}{$\ldots \ldots \ldots \ldots \ldots \ldots \ldots, \mathrm{mm}, \ldots \ldots \ldots \ldots \ldots \ldots \ldots$} & $\ldots \ldots \% \ldots \ldots$ \\
\hline Out & 16,1 & 3,8 & 0,5 & 2,9 & 58 \\
\hline Nov & 15,9 & 3,9 & 3,3 & 2,9 & 66 \\
\hline Dez & 16,7 & 3,5 & 1,3 & 2,6 & 67 \\
\hline Jan & 17,1 & 4,2 & 2,7 & 3,2 & 69 \\
\hline Fev & 17,1 & 3,3 & 2,9 & 2,5 & 70 \\
\hline Mar & 16,6 & 3,7 & 4,3 & 2,8 & 72 \\
\hline Media & 16.6 & 3,7 & - & 2,8 & 67 \\
\hline
\end{tabular}

$\mathrm{T}=$ temperatura, $\mathrm{Ev}=$ evaporação, $\mathrm{Pp}=$ precipitação, $\mathrm{ETo}=$ evapotranspiração de referência, $\mathrm{HR}=$ humidade relativa. Fonte: Estação Meteorológica Augusto Weberbauer. SA-FCA/UNC (2016).

A área total do experimento foi de $256,5 \mathrm{~m}^{2}$, o solo foi classificado como Andossolo mólico, com textura argilosa, conteúdo médio de matéria orgânica $(2,5 \%)$, e pH $(6,0), \mathrm{P}\left(21,4 \mathrm{mg} \mathrm{kg}^{-1}\right)$ e K $(280,0 \mathrm{mg}$ $\mathrm{kg}^{-1}$ ). A adubação do solo foi com $7 \mathrm{t} \mathrm{ha}^{-1}$ de húmus de minhoca (A1), guano de ilha (A2) e húmus de esterco de porquinho-da-índia (A3) como observa-se na Tabela 2.

A semeadura foi direta, realizada em 19/10/2015, usando sementes de acelga da var. Gigante Fordhook, em espaçamento de $30 \times 30 \mathrm{~cm}$ entre plantas e fileiras, ocupando $216 \mathrm{~m}^{2}$ de área cultivada, com 2400 plantas (111.111 $\mathrm{pl} \mathrm{ha}^{-1}$ ), e 36 unidades experimentais de $6 \mathrm{~m}^{2}$ (66 plantas), com área útil de 1,26 $\mathrm{m}^{2}$ (14 plantas). As coberturas foram colocadas depois da semeadura, sendo $8 \mathrm{t} \mathrm{ha}^{-1}$ de serragem de pinheirode-folhas-pêndulas (C1), casca de arroz (C2) e palha de aveia (C3). A emergência ocorreu aos sete dias depois da semeadura (DDS).

As regas foram complementares às precipitações pluviais (Tabela 1), considerando as necessidades hídricas da cultura, em função da evapotranspiração de referência (ETo), determinado pelo Tanque Classe 
A da estação meteorológica Augusto Weberbauer do SA-FCA, segundo metodologia de DOORENBOS \& PRUITT (1977).

Tabela 2. Resultados das análises químicas de adubo de húmus de minhoca (A1), guano de ilha (A2), e húmus de esterco de porquinho-da-índia (A3).

Table 2. Results of chemical analysis from worm humus fertilizer (A1), island guano (A2), and guinea pig manure humus (A3).

\begin{tabular}{rccccccccc}
\hline \multirow{2}{*}{ Adubo } & $\mathrm{pH}$ & $\begin{array}{c}\mathrm{C} . \mathrm{E} \\
\mathrm{dS} \mathrm{m}^{-1}\end{array}$ & $\mathrm{M} . \mathrm{O}$ & $\mathrm{N}$ & $\mathrm{P}_{2} \mathrm{O}_{5}$ & $\mathrm{~K}_{2} \mathrm{O}$ & $\mathrm{CaO}$ & $\mathrm{MgO}$ & $\mathrm{Na}$ \\
\hline $\mathrm{A} 1$ & 8,50 & 4,79 & 34,66 & 1,37 & 0,45 & 1,11 & 5,79 & 0,56 & 0,30 \\
$\mathrm{~A} 2$ & 7,50 & 117,20 & 12,59 & 0,32 & 1,26 & 0,80 & 8,00 & 0,50 & 0,80 \\
$\mathrm{~A} 3$ & 7,31 & 15,20 & 64,30 & 1,80 & 0,82 & 5,54 & 4,21 & 1,59 & 0,38 \\
\hline
\end{tabular}

$\mathrm{pH}=$ por potenciômetro; $\mathrm{C}$.E = medição indireta; $\mathrm{M} . \mathrm{O}=$ método de Walkley e Black; $\mathrm{N}=$ método de Kjeldahl; $\mathrm{P}_{2} \mathrm{O}_{5}=$ método do azul de molibdênio; $\mathrm{K}_{2} \mathrm{O}$ e $\mathrm{CaO}$ = por gravimetria e; $\mathrm{Na}$ e $\mathrm{MgO}=$ espectrofotometria de absorção atómica. Fonte: Laboratório de solos, plantas, água e fertilizantes. UNALM, 2016.

Realizaram-se três capinas manuais, aos 30, 94 e 115 DDS, também, realizou-se controle fitossanitário de Chaetocnema sp. e Diabrotica spp., com bioextractos de frutos de Capsicum pubescens (60\%) e bulbos de Allium sativum (40\%) em doses de $2 \mathrm{ml} \mathrm{L}^{-1}$.

Quando as folhas alcançaram a maturidade comercial (tamanho médio de $40 \mathrm{~cm}$, suculentas de cor verdes-escuros com pecíolos brancos), se realizaram colheitas aos 93 (1a), $114\left(2^{\mathrm{a}}\right)$ e $144\left(3^{\mathrm{a}}\right)$ DDS, respectivamente.

As avaliações da porcentagem de emergência (E) foram feitas por contagem até o dia sete DDS. Em cada colheita avaliou-se o comprimento de folhas (CF) utilizando uma régua milimétrica; massa fresca de folhas (MFF) e massa seca de folhas (MSF) pesadas em balança com precisão 0,01 g. Para determinar a MSF as folhas foram secas em estufa a $105^{\circ} \mathrm{C}$ até obter peso constante.

O delineamento experimental foi de blocos casualizados, com arranjo fatorial $3 \times 3$ (coberturas e adubos), em parcelas subdivididas (colheitas), com quatro repetições. Os dados foram submetidos a testes de normalidade e homogeneidade, para realizar a análise de variância, determinado a significância de tratamentos pelo teste $F$. As comparações múltiplas de médias foram feitas pelo teste de Tukey $(p<0,05)$ usando o software SISVAR.

As condições climáticas (Tabela 1), os adubos (Tabela 2) e as coberturas proporcionaram produtividades totais na $1^{\mathrm{a}}, 2^{\mathrm{a}}$ e $3^{\mathrm{a}}$ colheita de acelga de 24,$7 ; 27,8$ e $15,4 \mathrm{t} \mathrm{ha}^{-1}$, respectivamente, constituindo para o ciclo produtivo 67,9 $\mathrm{t} \mathrm{ha}^{-1}$. Em outras variedades, JOVER (2009) obteve produtividade de $26,4 \mathrm{t} \mathrm{ha}^{-1}$ (var. Penca verde) e 17,4 t ha ${ }^{-1}$ (var. Penca blanca) para uma só colheita; entretanto, LÓPEZPÉREZ et al. (2003), em casa de vegetação com adubação orgânica de $50 \mathrm{t} \mathrm{ha}^{-1}$, em dois anos consecutivos obtiveram rendimentos de 155 e $214 \mathrm{t} \mathrm{ha}^{-1}$.

De acordo com a análise de variância não houve efeito da interação entre os fatores cobertura e adubação para a característica avaliada. Por outro lado, as coberturas independentes dos adubos diferem nas variáveis CF e MFF, mas os adubos independentes das coberturas diferem na E, CF, MFF e MSF; por sua vez, as colheitas diferem no CF e MSF, existindo interação entre adubos e colheitas para a MFF. Os testes de médias são observados na Tabela 3 e Tabela 4.

A velocidade de decomposição das coberturas varia pincipalmente em função de sua relação $\mathrm{C} / \mathrm{N}$, consequentemente a incorporação de nutrientes ao solo é variado no tempo. Os valores de CF e MFF foram maiores com as coberturas C2 e C3 que quando utilizou-se C1 (Tabela 3). Resultados similares obtiveram RESENDE et al. (2005) em cenoura quando determinaram que a cobertura de C2 proporcionou maiores valores que a cobertura C1. Segundo GODOY et al. (2009), as coberturas de origem de gramíneas são de rápida degradação microbiológica por sua baixa relação $\mathrm{C} / \mathrm{N}(29 / 1)$, contrariamente à serragem de madeira, que tem alto teor de celulose, hemicelulose e lignina; CARRIJO et al. (2004) determinaram para C1 e C2 relações de $\mathrm{C} / \mathrm{N}$ de 156,5 e 50,0, respectivamente. Por sua parte DONEDA et al. (2012) determinaram para C3 uma relação $\mathrm{C} / \mathrm{N}$ de 22,8. A influência favorável das coberturas de C2 e C3 no CF e MFF da acelga, provavelmente, foi por sua baixa relação de $\mathrm{C} / \mathrm{N}$ que lhes permitiram maior decomposição e maior aporte de $\mathrm{N}$ à cultura. No entanto, MURGA-ORRILLO et al. (2016a \& 2016b), usando cobertura de palha de milho, demonstraram maior produção por uma maior dotação e eficiência de uso de água nas culturas de milho e feijão-caupi. 
Tabela 3. Médias de emergência (E), comprimento de folha (CF), massa fresca de folhas (MFF) e massa seca de folhas (MSF) de acelga em função do material de cobertura do solo, do adubo orgânico e da colheita. SA-FCA/UNC. Cajamarca, 2015/2016.

Table 3. Means of emergence (E), leaf length (CF), fresh leaf mass (MFF) and leaf dry mass (MSF) of Swiss chard as a function of soil cover material, organic fertilizer and harvest. SA-FCA / UNC. Cajamarca, 2015/2016.

\begin{tabular}{|c|c|c|c|c|c|c|c|c|c|c|c|}
\hline \multirow[b]{2}{*}{ Cobertura } & $E$ & $\mathrm{CF}$ & MFF & MSF & \multirow[b]{2}{*}{ Adubo } & $E$ & $\mathrm{CF}$ & MSF & \multirow[b]{2}{*}{ Colheita } & $\mathrm{CF}$ & MSF \\
\hline & $\%$ & $\mathrm{~cm}$ & \multicolumn{2}{|c|}{$\ldots . . .9 \mathrm{pl}^{-1} \ldots .}$. & & $\%$ & $\mathrm{~cm}$ & $\mathrm{~g} \mathrm{pl}^{-1}$ & & $\mathrm{~cm}$ & $\mathrm{~g} \mathrm{pl}^{-1}$ \\
\hline $\mathrm{C} 1$ & $88,2 a$ & $40,2 b$ & $179,4 b$ & $12,9 a$ & A1 & $83,7 b$ & $40,3 b$ & $12,7 b$ & $1^{\mathrm{a}}$ & $42,1 b$ & $14,5 a$ \\
\hline C2 & $87,7 a$ & $43,1 a$ & $209,9 a$ & $14,4 a$ & A2 & $85,4 b$ & $41,5 b$ & $13,8 a b$ & $2^{a}$ & $47,0 a$ & $16,9 a$ \\
\hline C3 & $85,1 \mathrm{a}$ & $42,7 a$ & $215,5 a$ & $14,6 a$ & A3 & $92,0 \mathrm{a}$ & $44,2 a$ & $15,4 a$ & $3^{a}$ & $36,9 c$ & $10,5 b$ \\
\hline
\end{tabular}

$\mathrm{C} 1$ = serragem de pinheiro-de-folhas-pêndulas, $\mathrm{C} 2$ = casca de arroz, $\mathrm{C} 3$ = palha de aveia, $\mathrm{A} 1$ = húmus de minhoca, $\mathrm{A} 2$ = guano de ilha, e A3 = húmus de esterco de porquinho-da-índia. Médias com mesma letra na coluna não diferem pelo teste de Tukey $(p>0,05)$.

Observa-se que o adubo A3 proporcionou maiores valores de E, CF e MSF que quando usado os adubos A1 e A2 (Tabela 3). O melhor desempenho do adubo A3 na acelga associasse-se, provavelmente, ao seu maior conteúdo de $\mathrm{K}$ e N em relação aos adubos $\mathrm{A} 1$ e $\mathrm{A} 2$ (Tabela 2). Outras pesquisas também obtiveram melhores resultados no MFF e MSF em alface e repolho (RODRIGUES \& CASALI 1998, FERREIRA et al. 2013, MUÑOZ et al. 2015), relacionando-os ao conteúdo de $\mathrm{N}$ dos adubos orgânicos.

Tabela 4. Massa fresca de folhas (MFF) nas três colheitas de acelga em função de adubos orgânicos, no SAFCA/UNC. Cajamarca, 2015/2016.

Table 4. Fresh leaf mass (MFF) in three harvests of Swiss chard as a function of organic fertilizers in SAFCA/UNC. Cajamarca, 2015/2016.

\begin{tabular}{cccc}
\hline Adubo & \multicolumn{2}{c}{ MFF $\left(\mathrm{kg} \mathrm{pl}^{-1}\right)$} & $3^{\mathrm{a}}$ \\
\hline A1 & $1^{\mathrm{a}}$ & $2^{\mathrm{a}}$ & $0,122 \mathrm{bA}$ \\
$\mathrm{A} 2$ & $0,189 \mathrm{aB}$ & $0,222 \mathrm{aA}$ & $0,133 \mathrm{cA}$ \\
A3 & $0,206 \mathrm{bB}$ & $0,250 \mathrm{aA}$ & $0,156 \mathrm{bA}$ \\
\hline
\end{tabular}

A1 = húmus de minhoca, $\mathrm{A} 2$ = guano de ilha, e A3 = húmus de esterco de porquinho-da-índia. Médias com mesma letra minúscula nas linhas e maiúscula nas colunas não diferem entre si pelo teste de Tukey $(p>0,05)$.

Nas colheitas da acelga, o CF foi maior na $2^{a}$, médio na $1^{\text {a }}$ e menor na $3^{a}$ colheita; entretanto, a MSF foi maior na $1^{\mathrm{a}}$ e $2^{\mathrm{a}}$ colheitas (Tabela 3 ). Para MFF (Tabela 4), de acordo com a interação, na $1^{\mathrm{a}}$ colheita, 0 adubo A3 proporcionou valor superior aos de $\mathrm{A} 2$ e $\mathrm{A} 1$. Na $2^{\underline{a}}$ e $3^{\mathrm{a}}$ colheitas, não mostraram diferenças significativas entre si. O adubo A3 destaca-se na produção de acelga. GARFÍ et al. (2011) mostraram bons resultados do $\mathrm{A} 3$, na forma líquida (biofertilizante), na produção de batata, com incremento de $100 \%$ na produtividade frente à testemunha.

Os adubos $A 1$ e $A 3$ na $1^{\text {a }}$ e $2^{\text {a }}$ colheita proporcionaram maiores MFF que na $3^{\text {a }}$ colheita; mas, com o adubo A2, a maior MFF foi na $2^{a}$ colheita, com valor médio na $1^{\text {a }}$ colheita e o menor valor na $3^{\text {a }}$ colheita (Tabela 4). Resultados similares determinaram ZIECH et al. (2014) e HERNÁNDEZ et al. (2016) em alface com uma só adubação orgânica para duas safras sucessivas, obtiveram maior disponibilidade de nutrientes para a $2^{a}$ colheita com resultados maiores de MFF que na $1^{\text {a }}$ colheita. O menor valor da MFF na $3^{\text {a }}$ colheita da acelga pode ser devido a fatores como baixa disponibilidade de nutrientes no solo por terem sido absorvidos na $1^{\underline{a}}$ e $2^{\underline{a}}$ colheitas, por lixiviação e/ou volatilização, por condições climáticas e idade da cultura. Em abobrinha-italiana, as variações entre colheitas foram atribuídas às condições climáticas e ao tipo de manejo (LÚCIO et al. 2008, CASTOLDI et al 2009). Em couve-flor, determinaram maior absorção de N e K, em consequência maior produção de fotoassimilados na fase inicial; entretanto, DAY et al. (2001) determinam diminuição da eficiência fisiológica e tamanho de folhas em função da idade das plantas.

A aplicação de coberturas ao solo de casca de arroz e palha de aveia, incrementam o comprimento e peso das folhas. Entretanto o húmus de esterco de porquinho-da-índia, favorece a germinação, o comprimento das folhas, e incrementa a massa fresca e seca da acelga. 
Os rendimentos da acelga, com húmus de minhoca, guano de ilha, e húmus de esterco de porquinhoda-índia mantiveram-se na $1^{\text {a }}$ e $2^{a}$ colheita, baixando significativamente na $3^{a}$ colheita.

O húmus de esterco de porquinho-da-índia apresenta caraterísticas nutricionais destacáveis para seu uso na horticultura.

\section{REFERÊNCIAS}

CARRIJO OA et al. 2004. Produtividade do tomateiro em diferentes substratos e modelos de casas de vegetação. Horticultura Brasileira 22: 5-9.

CASTOLDI R et al. 2009. Crescimento, acúmulo de nutrientes e produtividade da cultura da couve-flor. Horticultura Brasileira 27: 438-446.

COMESE RV et al. 2009. Cambios en las propiedades de suelo de huerta y rendimiento de Beta vulgaris var. cicla (L) por el uso de enmiendas orgánicas. Ciencia del Suelo 27: 271-275.

COSTA DMA et al. 2007. Eficiência da cobertura morta na retenção de umidade no solo. Holos 23: 59-69.

DAY ME et al. 2001. Age-related changes in foliar morphology and physiology in red spruce and their influence on declining photosynthetic rates and productivity with tree age. Tree Physiology 21: 1195-1204.

DONEDA A et al. 2012. Fitomassa e decomposição de resíduos de plantas de cobertura puras e consorciadas. Revista Brasileira de Ciência do Solo 36: 1714-1723.

DOORENBOS J \& PRUITT WO. 1977. Crop water requirements. 2.ed. Rome: FAO. 179p. (FAO Irrigation and Drainage Paper 24).

DZIDA K \& PITURA K. 2008. The influence of varied nitrogen fertilization on yield and chemical composition of Swiss chard (Beta vulgaris var. cicla L.). Acta Scientiarum Polonorum Hortorum Cultus 7: 15-24.

FERREIRA ICPV et al. 2013. Cobertura morta e adubação orgânica na produção de alface e supressão de plantas daninhas. Revista Ceres 60: 582-588.

GARFí $M$ et al. 2011. Psychrophilic anaerobic digestion of guinea pig manure in low-cost tubular digesters at high altitude. Bioresource Technology 102: 6356-6359.

GODOY JRR et al. 2009. Vermicompostagem de biossólido obtido de fossas sanitárias, grama e pó de serragem utilizando Eisenia foetida (Savigny, 1826). Revista Ceres 56: 648-653.

GONÇALVES AO et al. 2005. Efeitos da cobertura do solo com filme de polietileno azul no consumo de água da cultura da alface cultivada em estufa. Engenharia Agrícola Jaboticabal 25: 622-631.

HERNÁNDEZ T et al. 2016. Use of compost as an alternative to conventional inorganic fertilizers in intensive lettuce (Lactuca sativa L.) crops-Effects on soil and plant. Soil \& Tillage Research 160: 14-22.

JOVER PL. 2009. Verduras de hoja. Información para Extensión. Colonia Benítez: INTA-EEA. 13p.

KOŁOTA E et al. 2010. Yield and nutritional value of Swiss chard grown for summer and autumn harvest. Journal of Agricultural Science 2: 120-124.

KÖPPEN W. 1931. Grundriss der Klimakunde: Outline of climate science. Berlin: Walter de Gruyter. 388p.

LÓPEZ-PÉREZ JA et al. 2003. Alternativas al bromuro de metilo en cultivos protegidos de la Comunidad de Madrid. Boletín de Sanidad Vegetal 29: 481-489.

LÚCIO AD et al. 2008. Variância e média da massa de frutos de abobrinha-italiana em múltiplas colheitas. Horticultura Brasileira 26: 335-341.

MUÑOZ CRM et al. 2015. Evaluación de abonos orgánicos utilizando como indicadores plantas de lechuga y repollo en Popayan, Cauca. Biotecnología en el Sector Agropecuario y Agroindustrial 13: 73-82.

MURGA-ORRILLO $\mathrm{H}$ et al. 2016a. Evapotranspiração e coeficiente de cultivo do feijão-caupi cultivado em solo do cerrado submetido à cobertura morta. Revista Irriga 21: 172-187.

MURGA-ORRILLO H et al. 2016b. Influência da cobertura morta na evapotranspiração, coeficiente de cultivo e eficiência de uso de água do milho cultivado em cerrado. Revista Irriga 21: 352-364

RESENDE FV et al. 2005. Uso de cobertura morta vegetal no controle da umidade e temperatura do solo, na incidência de plantas invasoras e na produção da cenoura em cultivo de verão. Ciência e Agrotecnologia 29: 100-105.

RODRIGUES ET \& CASALI VWD. 1998. Resposta da alface à adubação orgânica. II. Teores, conteúdos e utilização de macronutrientes em cultivares. Revista Ceres 45: 437-449.

XIUKANG W et al. 2015. Effects of mulching and nitrogen on soil temperature, water content, nitrate-N content and maize yield in the Loess Plateau of China. Agricultural Water Management 161: 53-64.

ZIECH ARD et al. 2014. Cultivo de alface em diferentes manejos de cobertura do solo e fontes de adubação. Revista Brasileira de Engenharia Agrícola e Ambiental 18: 948-954. 